\title{
Phenomenological theory of Superconductivity in the frameworks of QCD and RCD
}

\author{
Balwant S. Rajput \\ I-11, Gamma-2, Greater Noida, India \\ Email: bsrajp@gmail.com
}

\begin{abstract}
Carrying out the study of phenomenological theory of superconductivity at microscopic level in the framework of QCD and restricted chromodynamics, we have investigated the topology of dyons in magnetic gauge space and showed that RCD vavuum containing plasma of dyons and anti-dyons behave like a chromomagnetic superconductor within which the chromomagnetic gluons propagate in the non-perturbative vacuum. Study of the superconductivity, dual superconductivity and color superconductivity have shown to automatically incorporate the condensation of monopoles or dyons ultimately leading to superconducting confining phases of the QCD vacuum.
\end{abstract}

Keywords: QCD, Dual superconductivity and confinement, Monopoles and Dyons.

\section{Introduction}

In the process of current understanding of superconductivity, in a series of papers [1-9] we have conceived its hopeful analogy with QCD and demonstrated that the essential features of superconductivity i.e., the Meissner effect and flux quantization, provide the vivid models for actual confinement mechanism in QCD. The condensation of magnetic monopole incorporates the state of magnetic superconductivity and the notion of chromo magnetic superconductor [10] where the Meissner effect confining magnetic field in ordinary superconductivity would be replaced by the chromo-electric Meissner effect (the dual Meissner effect), which would confine the color electric flux. However, the crucial ingredient for condensation in a chromo-magnetic superconductor would be the non-Abelian force in contrast to the Abelian ones in ordinary superconductivity. Topologically, a nonAbelian gauge theory is equivalent to a set of Abelian gauge theories supplemented by monopoles. The method of Abelian projection $[11,12]$ is one of the popular approaches to confinement problem, together with dual superconductivity picture, in non-Abelian gauge theories.

\section{Elecromagnetic Duality and Dyonic Interactions}

A gauge invariant and Lorentz covariant quantum field theory of fields associated with dyons has been developed [13-16] in purely group theoretical manner by using two fourpotentials and assuming the generalized charge, generalized current and generalized fourpotential as complex quantities with their real and imaginary parts as electric and magnetic constituents i.e.

The generalized charge, four current and fourpotential are given as, 
$\mathrm{q}=\mathrm{e}-\mathrm{ig}$

$J_{\mu}=j_{\mu}-i k_{\mu}$

$V_{\mu}=A_{\mu}-i B_{\mu}$

$\vec{\nabla} \cdot \vec{\Psi}=J_{0} \quad \vec{\nabla} \times \vec{\Psi}=-i \vec{J}-i \frac{\partial \vec{\Psi}}{\partial t}$

which gives

$G_{\mu \nu, v}=J_{\mu} ; G_{\mu \nu, v}^{d}=0$

where $G_{\mu \nu}=\partial_{\mu} V_{\nu}-\partial_{\nu} V_{\mu}$;

$$
G_{\mu \nu}^{d}=\frac{1}{2} \varepsilon_{\mu \nu \alpha \beta} G_{\alpha \beta}
$$

$$
\begin{array}{ll}
\text { It } \quad \text { implies } & G_{\mu \nu}=F_{\mu \nu}-i H_{\mu \nu} ; \\
F_{\mu \nu}=\partial_{\mu} A_{\nu}-\partial_{\nu} A_{\mu} ; & H_{\mu \nu}=\partial_{\mu} B_{v}-\partial_{\nu} B_{\mu} \\
\text { or } \quad F_{\mu v, v}=j_{\mu} ; & H_{\mu v, v}=k_{\mu}
\end{array}
$$

which are symmetrical under the duality transformations:

$$
\begin{aligned}
& F_{\mu \nu} \rightarrow H_{\mu \nu} ; H_{\mu \nu} \rightarrow-F_{\mu \nu} ; \\
& j_{\mu} \rightarrow k_{\mu} ; k_{\mu} \rightarrow-j_{\mu}
\end{aligned}
$$

For spin-1 generalized charge (i.e. bosonic dyon) of rest mass $m_{0}$ in Abelian Theory we have,

$$
\begin{aligned}
& L=m_{0}-\frac{1}{4}\left[\alpha\left\{\left(A_{v, \mu}-A_{\mu, v}\right)^{2}-\left(B_{v, \mu}-B_{\mu, v}\right)^{2}\right\}-\right. \\
& 2 \beta\left\{\left(A_{v, \mu}-A_{\mu, v}\right)\left(B^{v, \mu}-B^{\mu, v}\right)\right\} \\
& \left.+\left\{\left(\alpha A_{\mu}-\beta B_{\mu}\right) j^{\mu}-\left(\alpha B_{\mu}+\beta A_{\mu}\right) k^{\mu}\right\}\right] \\
& \quad=L_{P}+L_{F}+L_{I}
\end{aligned}
$$

$\alpha$ and $\beta$ are real positive unimodular parameters i.e.

$$
|\alpha|^{2}+|\beta|^{2}=1
$$

The action integral: $\quad S=\int_{t_{1}}^{t_{2}} L d t=S_{P}+S_{F}+S_{I}$
$\vec{\psi}=\vec{E}-i \vec{H}$

and the generalized field equations are,

Varying the trajectory of particle without changing the field we get equation of motion : $m \ddot{x}_{\mu}=\operatorname{Re}\left(q * G_{\mu v}\right) u^{v}$,

Residual angular momentum : $\quad \vec{J}_{r e s}=\mu_{i j} \frac{\vec{r}}{r} ;$ $\mu_{i j}=e_{i} g_{j}-e_{j} g_{i}$

There are monopole field configurations in any non-Abelian gauge theory. The phase structure of any such theory can be probed by adding a scalar field (i.e. Higgs field) in the adjoint representation so long as it does not change the nature of flow of the coupling constant with energy. Topologically, a non-Abelian gauge theory is equivalent to a set of Abelian gauge theories supplemented by monopoles which undergo condensation leading to confinement. The non-Abelian confinement of dyonic charge is related to linear Abelian theory in a dyonic superconductor. The action for dyonic field in Abelian projection of QCD may be written as $^{[16]}$

$$
S=-\frac{1}{4} \int G_{\mu v}(x) \notin(x-y) G^{\mu v} d^{4} x d^{4} y+J_{\mu} V^{\mu}
$$

Generalized dielectric constant $\notin(x-y)=\in(x-y)-i \mu(x-y)$ with $\in(x-y)$ as ordinary dielectric constant and $\mu(x-y)$ as magnetic permeability such that,

$$
\int \in(x-y) \mu(x-z) d^{4} y=\delta(x-z)
$$

Generalized four-current couples to $V_{\mu}$, with the current-correlators given by 


$$
\begin{gathered}
\left\langle J_{\mu}\right\rangle=\frac{\delta S}{\delta V_{\mu}} \\
\left\langle j_{\mu}(x) J(y)\right\rangle=\frac{\delta^{2} S}{\delta V_{v}(x) \delta V_{\mu}(y)} \\
\left\langle J_{\mu}(x) J_{v}(y)\right\rangle=-\int \frac{d^{4} k}{(2 \pi)^{4}} e^{i k(x-y)}\left[k^{2} \delta_{\mu v}-k_{\mu} k_{v}\right] \\
\notin\left(k^{2}\right)
\end{gathered}
$$

where $\notin\left(k^{2}\right)$ is Fourier transform of $\notin(x-y)$. For free fields in vacuum, $\notin\left(k^{2}\right)=1$. For perturbatively small $\notin\left(k^{2}\right)=1+\chi\left(k^{2}\right)$; $\chi\left(k^{2}\right)=\chi_{e}\left(k^{2}\right)-i \chi_{g}\left(k^{2}\right)$ with $\chi_{e}\left(k^{2}\right)$ as perturbation related with electric charge loop and $\chi_{g}\left(k^{2}\right)$ as the perturbation related with magnetic charge loop. With the generalized Meissner effect with its real and imaginary constituents as the strict Meissner effect and dual Meissner effect respectively, the generalized field $V_{\mu}$ can penetrate in to a generalized superconductor up to the generalized London penetration depth

$$
\lambda_{L}=\lambda_{e}-i \lambda_{g}
$$

For small values of $k^{2}$,

$$
\begin{aligned}
& \notin\left(k^{2}\right)=\frac{m_{L}^{2}}{k^{2}}-\frac{i k^{2}}{m_{L}^{2}} ; \\
& m_{L}=\frac{1}{\lambda_{L}}=m_{l_{e}}-i m_{L_{g}}
\end{aligned}
$$

With,

$$
m_{L_{e}}=\frac{\lambda_{e}}{\left|\lambda_{L}\right|^{2}} ; m_{L_{g}}=-\frac{\lambda_{g}}{\left|\lambda_{L}\right|^{2}}
$$

which gives,

$$
\begin{aligned}
& \left\langle\left[j_{\mu}(x) j_{v}(y)+k_{\mu}(x) k_{v}(y)\right]\right\rangle=-\int \frac{d^{4} k}{(2 \pi)^{4}} e^{i k(x-y)} \\
& {\left[k^{2} \delta_{\mu v}-k_{\mu} k_{v}\right] \in\left(k^{2}\right)} \\
& \left\langle\left[j_{\mu}(x) k_{\mu}(y)-j_{v}(x) k_{\mu}(y)\right]\right\rangle=-\int \frac{d^{4} k}{(2 \pi)^{4}} e^{i k(x-y)} \\
& {\left[k^{4} \delta_{\mu v}-k^{2} k_{\mu} k_{v}\right] \mu\left(k^{2}\right) / m_{L}{ }^{2}}
\end{aligned}
$$

For electric and magnetic charges on different particles (i.e. not dyons);

$$
F_{\mu v, v}=j_{\mu} \text {; }
$$

$F_{\mu v, v}^{d}=0$;

$H_{\mu v, v}=k_{\mu} ; \quad \Rightarrow \square \mathrm{A}_{\mu}=\mathrm{j}_{\mu} ; \square \mathrm{B}_{\mu}=k_{\mu} ;$

$H_{\mu v, v}^{d}=0$

$$
\begin{aligned}
& m \ddot{x}_{\mu}=\left(e F_{\mu v}+g H_{\mu v}\right) u^{v} ; \\
& S=-\frac{1}{4} \int F_{\mu v}(x) \in(x-y) F^{\mu v}(y) d^{4} x d^{4} y \\
& -\frac{1}{4} \int H_{\mu v}(x) \mu(x-y) H^{\mu v}(y) d^{4} x d^{4} y+ \\
& j_{\mu} A^{\mu}+k_{\mu} B^{\mu}
\end{aligned}
$$$$
\left\langle j_{\mu}\right\rangle=\frac{\delta S}{\delta A_{\mu}} ;\left\langle k_{\mu}\right\rangle=\frac{\delta S}{\delta B_{\mu}} ;
$$$$
\left\langle j_{\mu}(x) j_{v}(y)\right\rangle=\frac{\delta^{2} S}{\delta A_{v}(y) \delta A_{\mu}(x)} ;
$$$$
\left\langle k_{\mu}(x) k_{v}(y)\right\rangle=\frac{\delta^{2} S}{\delta B_{v}(y) \delta B_{\mu}(x)}
$$$$
\Rightarrow
$$$$
\left\langle j_{\mu}(x) j_{v}(y)\right\rangle=-\int \frac{d^{4} k}{(2 \pi)^{4}}\left[\delta_{\mu v}-\frac{k_{\mu} k_{v}}{k^{2}}\right] m_{L_{e}}{ }^{2}
$$

and

$$
\left\langle k_{\mu}(x) k_{v}(y)\right\rangle=-\int \frac{d^{4} k}{(2 \pi)^{4}}\left[\delta_{\mu v}-\frac{k_{\mu} k_{v}}{k^{2}}\right] m_{L_{g}}{ }^{2}
$$

which show that:

Charged particles $\left[\chi_{e}\left(k^{2}\right) \geq 1\right]$ produce screening effect for the $A_{\mu}$-propagator, with 
the corresponding photon acquiring the mass $m_{L_{e}}$ and anti-screening effect for the $B_{\mu}$ - propagator. The monopole loops produce screening effect for $B_{\mu}$-propagator, with corresponding photon acquiring the mass $m_{L_{g},}$, and anti-screening effect for $A_{\mu}$-propagator. Thus any particle ( electrically charged or a monopole) screens its own direct potential to which it minimally couples, and anti-screens the dual potential ( $B_{\mu}$ for electric charge and $A_{\mu}$ for monopole).This dual anti-screening effect leads to dual superconductivity in accordance with generalized Miessner effect.

\section{Superconductivity due to condensation and confinement of dyons}

Suitable Lagrangian density of a spontaneously broken non-Abelian gauge theory SU(2), yielding the classical dyonic solutions has been obtained as

$$
\begin{aligned}
& L=-\frac{1}{4} G_{\mu \nu}^{a} G_{a}^{\mu \nu}+\frac{1}{2}\left(D_{\mu} \phi\right)^{a}\left(D^{\mu} \phi\right)_{a}-V(\phi)= \\
& L_{d y o n}\left(A_{\mu}, B_{\mu}, \phi\right) \\
& D_{\mu} \phi=\partial_{\mu} \phi-i \operatorname{Re}\left(q * V_{\mu}\right) \phi=\left(\partial_{\mu}-i e A_{\mu}-i g B_{\mu}\right) \phi \\
& V(\phi)=\frac{1}{4}\left(\phi^{a} \phi_{a}\right)^{2}-\frac{1}{2} v^{2}\left(\phi^{a} \phi_{a}\right)
\end{aligned}
$$

with

$$
v=\langle\phi\rangle=\langle 0|\phi| 0\rangle
$$

Gauge dependent part of Lagrangian ( first term of rhs) is invariant under transformatio $\mathrm{n}$ with

$$
\delta=\tan ^{-1}\left(\frac{g}{e}\right)
$$

Using this Lagrangian density the electric and magnetic fields of dyons have been obtained as,
$E^{a}{ }_{i}=G^{a}{ }_{0 i}=\partial^{i} V^{a}{ }_{0}+|q| \varepsilon^{a b c} V_{i b} V_{0 c}=\left(D_{i} \phi\right)^{a} \sin \alpha$,

$B^{a}{ }_{i}=\varepsilon_{i j k} G^{j k a}=\left(D_{i} \phi\right)^{a} \cos \alpha$

$D_{0}(\phi)^{a}=0 ; \alpha=\tan ^{-1} \frac{e}{g}$

and dyonic mass $M=v\left(e^{2}+g^{2}\right)^{\frac{1}{2}}=v|q|$

Under Abelian projection we have [17]

$$
\begin{aligned}
E_{j}{ }^{a} & =-\frac{3 b}{|q| r^{4}}(\vec{r})^{a}(\vec{r})_{j}-\frac{2 c}{|q| r^{3}}(\vec{r})^{a}(\vec{r})_{j} ; \\
B_{j}{ }^{a} & =-\frac{(\vec{r})_{j}(\vec{r})^{a}}{|q| r^{4}}
\end{aligned}
$$

For vanishing $\mathrm{c}$ these fields corresponds to point-like mass-less dyons with electric charge $\frac{3 b}{|q|}$ and magnetic charge $\frac{1}{|q|}$. Thus non-Abelian dyons give rise to the Abelian dyons in the Abelian projection. The infra-red properties of QCD in the Abelian projection can be described by the following Lagrangian density in the Abelian Higgs Model (AHM) [17] in which dyons are condensed.

$$
\begin{aligned}
& L_{\text {dyon }}\left(A_{\mu}, B_{\mu}, \phi\right)=-\frac{1}{4} G_{\mu \nu} G^{\mu v} \\
& +\frac{1}{2}\left|\left(\partial_{\mu}-i e A_{\mu}-i g B_{\mu}\right) \phi\right|^{2}+\eta\left(|\phi|^{2}-v^{2}\right)^{2}
\end{aligned}
$$

Partition function in Euclidean space-time is

$Z_{d y o n}=\int D A_{\mu} D B_{\mu} D \phi \exp \left\{-\int d^{4} x L_{d y o n}\left(A_{\mu}, B_{\mu}, \phi\right)\right\}$

Applying transformation $\left(A_{\mu}, B_{\mu}\right) \rightarrow\left(A_{\mu}^{\prime}, B_{\mu}^{\prime}\right)$ and integrating over the field $A_{\mu}^{\prime}$ we get

$$
\begin{aligned}
& Z_{d y o n}=\int D B_{\mu}^{\prime} D \phi \exp \left\{-\int d^{4} x L_{A H M}\left(B_{\mu}^{\prime}, \phi\right)\right\} \\
& L_{A H M}\left(B_{\mu}^{\prime}, \phi\right)=-\frac{1}{4} H_{\mu v}^{\prime} H^{\prime \mu v}+\frac{1}{2}\left|\left(\partial_{\mu}-i \bar{g} B_{\mu}^{\prime}\right) \phi\right|^{2} \\
& +\eta\left(|\phi|^{2}-v^{2}\right)^{2}
\end{aligned}
$$


where $\quad \bar{g}=|q| ; H_{\mu \nu}^{\prime}=\partial_{\mu} B_{\nu}^{\prime}-\partial_{\nu} B_{\mu}^{\prime}$

This model (AHM) incorporates dual superconductivity and hence confinement as the consequence of dyonic condensation since the Higgs type mechanism arises here. Using the idea of confinement of electric flux due to condensation of magnetic monopoles, a dual gauge theory called restricted chromo dynamics (RCD) has been constructed [1] out of QCD in SU(2) theory. This dual gauge theory incorporates a dynamical dyonic eissner effect). In the Abelian Higgs model of RCD the mass of dual gauge boson is given by,

$$
\mathrm{M}_{\mathrm{D}=\mathrm{qv}}
$$

where $\mathrm{v}$ is the vacuum expectation value of dyonic field $\phi$ with charge $\mathrm{q}$ and mass

$$
\mathrm{M}_{\phi}=\sqrt{ }(8 \eta) \mathrm{v}
$$

With these two mass scales the coherence length $\varepsilon$ and the penetration length $\lambda$ are given by $\varepsilon=1 / \mathrm{M}_{\phi}=1 /[\sqrt{ }(8 \eta) \mathrm{v}] ; \lambda=1 / \mathrm{M}_{\mathrm{D}}=1 /(|\mathrm{q}| \mathrm{v})$ . In the confinement phase of RCD the dyons are condensed. The region in phase space, where $\varepsilon=\lambda$, constitutes the border between type-I and type-II super-conductors. Thus dyonically condensed vacuum is characterized by the presence of two massive modes. The mass of the scalar mode, $\mathbf{M}_{\phi}$ determines how fast the perturbative vacuum around a colored source reaches condensation and the mass $\mathrm{M}_{\mathrm{D}}$ of vector mode determines the penetration length of the colored flux. We have also undertaken $[18,19]$ the study of behavior of Dyons around the RCD String and showed that magnetic constituent of dyonic current at large transverse distance from the string should be controlled by the coherence length and the penetration length where the coherence length could be derived directly from the measurement of dyonic density around a chromo-dyonic flux condensation and exhibits the desired dual dynamics that guarantees the confinement of dyonic quark through generalized Meissner effect. Here we get dyonic super current, the real part of which (electric constituent) screens the electric flux which confines the magnetic color charge (through usual Meissner effect) and the imaginary part (i.e. magnetic constituent) of this super-current screens the magnetic flux that confines the electric color iso- charges (due to dual $M$ spanned between a static quark- anti- quark pair. In the maximal Abelian gauge, as used in RCD here, the penetration length and coherence length are almost the same and hence the vacuum is nearly the border between type-I and type-II dual superconductors.

\section{References}

1. Rajput,B.S., Kumar, S., Swarup, R., Singh, B.: Int.J.Theor.Phys.48, 1766 (2009) 2. Rajput, B.S. and Kumar, S.: Adv. High Energy Phys. 2010, Id713659 (2010)

3. Raiput, B.S. and Kumar, S.: Adv. High Energy Phys. 2010, Id 768054 (2011)

4. Raiput, B.S. and Kumar, S.: Il Nuovo Cim. 125B, 1499 (2010)

5. Raiput, B.S. and Kumar, S.: Int. Journ. Theor. Phys. 50, 1342 (2011)

6. Raiput, B.S. and Kumar, S.: Eur. Phys. J. Plus 126, 22 (2011)

7. Raiput, B.S. and Kumar, S.: Int. Journ. Theor. Phys. 50, 371 (2011)

8. Raiput, B.S. and Kumar, S.: Int. Journ. Theor. Phys. 50, 2347 (2011)

9. Raiput, B.S. and Kumar, S.: Int. Journ. Theor. Phys. 50, 3054 (2011)

10.Nair,V.P.,Rosenweig,C.:

Phys.Rev.D31,401(1985) 
11.Alessandro,A.D'.,Elia,M.D'.,TagliacozzoL.:

Nucl.Phys.B774,168(2007)

12.Boyko,P.Yu.,Barnyakov,V.G.,Ilgenfritz,E.M.,Ko

valenko,A.V.,Martemyano,B.V.

Muller-

Preussker,M.,Polikarpov,M.I.,Veselov,A.I.:

Nucl.Phys.B756,71(2006)

13.Rajput,B.S.,Prakash,Om.:

Ind.J.Phys.50,929(1976)

14.Rajput,B.S.,Bhakuni,D.S.,Negi,O.P.S.:

Lett.Nuovo.Cim.34,50(1983)

15. Rajput, B.S.,Joshi,D.C.:Pramana.13, 637 (1979)

16.Rajput,B.S.,Bhakuni,D.S.,Negi,O.P.S.:

Lett.Nuovo.Cim.36,15(1983)

17. Rajput, B.S.: Int. Journ. Theor. Phys. 56 (2017)

18. Rajput, B.S.: Eur. Phys. J. Plus (2017)132:118

19. Rajput, B.S.: Open Access J. Phys. 1(1) (2017)

54-63

$* * * * * * *$ 